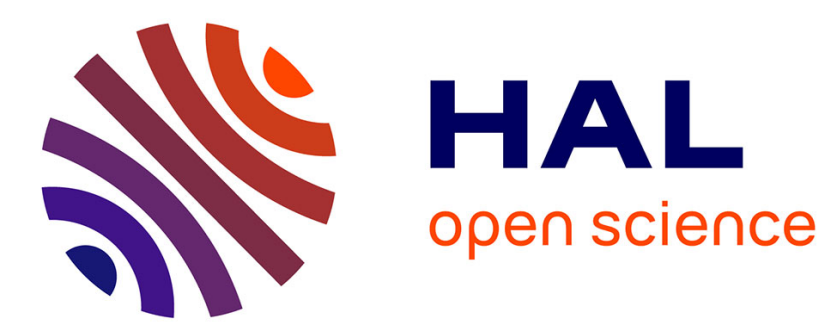

\title{
Asymptotic Detection Performance Analysis of the Robust Adaptive Normalized Matched Filter
}

\author{
Jean-Philippe Ovarlez, Frédéric Pascal, Arnaud Breloy
}

\section{To cite this version:}

Jean-Philippe Ovarlez, Frédéric Pascal, Arnaud Breloy. Asymptotic Detection Performance Analysis of the Robust Adaptive Normalized Matched Filter. IEEE 6th International Workshop on Computational Advances in Multi-Sensor Adaptive Processing (CAMSAP 2015), Dec 2015, Cancun, Mexico. 10.1109/CAMSAP.2015.7383755 . hal-01325083

\section{HAL Id: hal-01325083 \\ https://hal.science/hal-01325083}

Submitted on 27 Mar 2020

HAL is a multi-disciplinary open access archive for the deposit and dissemination of scientific research documents, whether they are published or not. The documents may come from teaching and research institutions in France or abroad, or from public or private research centers.
L'archive ouverte pluridisciplinaire HAL, est destinée au dépôt et à la diffusion de documents scientifiques de niveau recherche, publiés ou non, émanant des établissements d'enseignement et de recherche français ou étrangers, des laboratoires publics ou privés. 


\title{
Asymptotic Detection Performance Analysis of the Robust Adaptive Normalized Matched Filter
}

\author{
Jean-Philippe Ovarlez ${ }^{*}$, Frédéric Pascal ${ }^{\dagger}$ and Arnaud Breloy $\ddagger$ \\ * ONERA, DEMR/TSI, Chemin de la Hunière, 91120 Palaiseau, France and SONDRA/CentraleSupélec \\ (e-mail: jean-philippe.ovarlez@onera.fr) \\ † L2S/CentraleSupélec-CNRS-Université Paris-Sud, 3 rue Joliot-Curie, 91190 Gif-sur-Yvette, France \\ (e-mail: frederic.pascal@centralesupelec.fr) \\ $\ddagger$ SONDRA/CentraleSupélec, 3 rue Joliot-Curie, 91190 Gif-sur-Yvette, France \\ (e-mail: arnaud.breloy@centralesupelec.fr)
}

\begin{abstract}
This paper presents two different approaches to derive the asymptotic distributions of the robust Adaptive Normalized Matched Filter (ANMF) under both $H_{0}$ and $H_{1}$ hypotheses. More precisely, the ANMF has originally been derived under the assumption of partially homogenous Gaussian noise, i.e. where the variance is different between the observation under test and the set of secondary data. We propose in this work to relax the Gaussian hypothesis: we analyze the ANMF built with robust estimators, namely the $M$-estimators and the Tyler's estimator, under the Complex Elliptically Symmetric (CES) distributions framework. In this context, we analyse two asymptotic performance characterization of this robust ANMF. The first approach consists in exploiting the asymptotic distribution of the different covariance matrix estimators while the second approach is to directly exploit the asymptotic distribution of the ANMF distribution built with these estimates.
\end{abstract}

\section{INTRODUCTION}

In the general statistical signal processing area, the detection problem is an important topic of research [1], [2]. Since in practice, the noise parameters are unknown, an estimation step is required leading to the so-called adaptive detection processes. Among these unknown parameters, the noise covariance matrix is probably one of the most important since the resulting performance of adaptive detectors mainly relies on the its estimation accuracy. This is the case for the Adaptive Matched Filter (AMF) [3], the Kelly's test [4] and the Adaptive Normalized Matched Filter (ANMF) [5]. Generally, the covariance matrix is estimated thanks to the so-called Sample Covariance Matrix (SCM). Although this estimator is very simple and provides optimal performance under a Gaussian noise, the resulting adaptive detector performance can strongly be degraded when the noise turned to be non-Gaussian, heterogeneous or when it contains outliers/jammers.

To fill these gaps, a general framework on robust estimation theory has been extensively studied in the statistical community in the 1970s following the seminal works of Huber and Maronna [6], [7]. The multivariate real case has been recently extended to the complex case [8]-[10], more adapted for signal processing applications. Under this robust theory framework, most of recent works in covariance matrix estimation considers the broader class of Complex Elliptically Symmetric (CES) distributions. A complete review on CES applied to array processing can be found in [8].

In this CES framework, the so-called $M$-estimators [7] and the Tyler's estimator [10], [11] present alternatives to the Gaussianbased SCM. Although these robust estimators provide good results in practice [9], the statistical analysis of the resulting adaptive detectors is a difficult point. This is mainly due to the non explicit form of these estimators, defined through fixed point equations. However, their asymptotic properties have been recently derived in [8], [9]. Following these works, the aim of this paper is to derive the asymptotic properties of the ANMF built with these estimators, namely the $M$ estimators and the Tyler's estimator, under both $H_{0}$ (absence of target) and $H_{1}$ (presence of target) hypotheses. The interest of such an analysis is to provide a better statistical characterization of the ANMF than the one based on the Normalized Matched Filter (NMF) [12].

The paper is organized as follows: next section provides the general background of this work as well as a recall on the main results concerning the statistics of the NMF and ANMF detection tests under both Gaussian assumption and CES distributions background. Section III gives the results presented in [13] concerning the asymptotic distribution of the ANMF built with robust estimators. Then, Section IV presents two different ways to derive the statistic of the ANMF built with any M-estimators for both $H_{0}$ and $H_{1}$ hypotheses. Finally, some conclusions and perspectives are drawn in the last section.

\section{BACKGROUND}

A. The NMF and ANMF in partially homogeneous Gaussian environment

Detecting a complex signal corrupted by an additive Gaussian noise $\mathbf{c} \sim \mathcal{C} \mathcal{N}\left(\mathbf{0}, \sigma^{2} \mathbf{M}\right)$ in a $m$-dimensional complex observation vector $\mathbf{y}$ can be stated as the following binary hypothesis test:

$$
\left\{\begin{array}{lll}
H_{0}: \mathbf{y}=\mathbf{c} & \mathbf{y}_{i}=\mathbf{c}_{i} & i=1, \ldots, N \\
H_{1}: \mathbf{y}=\alpha \mathbf{p}+\mathbf{c} & \mathbf{y}_{i}=\mathbf{c}_{i} \quad i=1, \ldots, N
\end{array},\right.
$$

where $\mathbf{p}$ is a perfectly known complex steering vector, $\alpha$ is the unknown signal amplitude and where the $\mathbf{c}_{i} \sim \mathcal{C N}(\mathbf{0}, \mathbf{M})$ are $N$ signal-free independent measurements, traditionally called the secondary data, used to estimate the background covariance matrix $\mathbf{M}$. When $\mathbf{M}$ is known and the variance $\sigma^{2}$ is unknown, this binary hypothesis test is solved by the Generalized Likelihood Ratio Test (GLRT) theory leading to a well-known Normalized Matched Filter [12] denoted $H($.$) and defined on [0,1]$ by

$$
H(\mathbf{M})=\frac{\left|\mathbf{p}^{H} \mathbf{M}^{-1} \mathbf{y}\right|^{2}}{\left(\mathbf{p}^{H} \mathbf{M}^{-1} \mathbf{p}\right)\left(\mathbf{y}^{H} \mathbf{M}^{-1} \mathbf{y}\right)} .
$$

By denoting $\delta=\alpha^{2} \mathbf{p}^{H} \mathbf{M}^{-1} \mathbf{p} / \sigma^{2}$ the signal-to-noise ratio (SNR), the distribution of $H(\mathbf{M})$ derived for heterogeneous Gaussian under $H_{0}$ or $H_{1}$ hypotheses, environment, is given by:

$$
p_{H(\mathbf{M})}(u)=e^{-\delta} \beta_{1, m-1}(u)_{1} F_{1}(m, 1 ; u \delta),
$$

where ${ }_{1} F_{1}\left(\alpha_{1}, \alpha_{2} ;.\right)$ is the complex confluent hypergeometric function and where $\beta_{1, m-1}(u)=(m-1)(1-u)^{m-2} \mathbb{1}_{[0,1]}(u)$ is the PDF of the central beta distribution with degrees of freedom 1 and 
$m-1$. Thus, setting $\delta$ to zero simply leads to the $\beta(1, m-1)$ PDF for $H(\mathbf{M})$ under $H_{0}$ hypothesis. Hence, the theoretical relationship between the detection threshold $\lambda$ and the Probability of False Alarm (PFA) is defined as $P_{f a}=\mathbb{P}\left(H(\mathbf{M})>\lambda \mid H_{0}\right)=(1-\lambda)^{m-1}$. This relation will serve as a benchmark since it characterizes the case of a perfectly known covariance matrix for the detection test. When $\delta \neq 0$, the Probability of Detection $P_{d}=\mathbb{P}\left(H(\mathbf{M})>\lambda \mid H_{1}\right)$ for a given SNR $\delta$ and for a fixed value of the detection threshold $\lambda$ is given by:

$$
P_{d}=1-e^{-\delta} \int_{0}^{\lambda} \beta_{1, m-1}(u)_{1} F_{1}(m, 1 ; u \delta) d u .
$$

When an estimate $\widehat{\mathbf{M}}$ of $\mathbf{M}$ is plugged into the NMF (two-step GLRT), this results in the so-called ANMF or ACE (Adaptive Coherence Estimator) [2], [5]. Assuming that the SCM, defined as $\widehat{\mathbf{M}}_{S C M}=\frac{1}{N} \sum_{k=1}^{N} \mathbf{c}_{k} \mathbf{c}_{k}^{H}$ is used, Kraut et al. have shown in [14] that $H\left(\widehat{\mathbf{M}}_{S C M}\right)$ has the following distribution:

$$
\begin{aligned}
f_{H\left(\widehat{\mathbf{M}}_{S C M}\right)}(x) & =\int_{0}^{1} u^{N-m+1} \frac{(1-u)^{m-1}(1-x)^{N-m}}{(1-u x)^{N-m+2}} \\
& \times \frac{e^{-\delta}}{K}{ }_{1} F_{1}\left(N-m+2,1 ; \frac{\delta x(1-u)}{1-x u}\right) d u,
\end{aligned}
$$

where $K=\Gamma(N-m+1) \Gamma(m-1) / \Gamma(N+1)$. By setting $\delta=0$ in the previous equation, the resulting PDF $f_{H\left(\widehat{\mathbf{M}}_{S C M}\right)}$ of $H\left(\widehat{\mathbf{M}}_{S C M}\right)$ under $H_{0}$ hypothesis can be retrieved [15]:

$$
\begin{aligned}
f_{H\left(\widehat{\mathbf{M}}_{S C M}\right)}(x) & =\frac{(N-m+1)(m-1)}{N+1}(1-x)^{N-m} \\
& \times{ }_{2} F_{1}(N-m+2 ; N-m+2 ; N+2 ; x),
\end{aligned}
$$

where ${ }_{2} F_{1}($.$) is the hypergeometric function. The theoretical relation-$ ship between the detection threshold $\lambda$ and the Probability of False Alarm $P_{f a}=\mathbb{P}\left(H\left(\widehat{\mathbf{M}}_{S C M}\right)>\lambda \mid H_{0}\right)$ is defined as:

$$
P_{f a}=(1-\lambda)^{N-m+1}{ }_{2} F_{1}(N-m+2, N-m+1 ; N+1 ; \lambda),
$$

whereas the corresponding relationship between $P_{d}$ and the SNR $\delta$ for a fixed $P_{f a}$ leads to:

$$
\begin{array}{rl}
P_{d}=1-\int_{0}^{1} & d u \int_{0}^{\lambda} u^{N-m+1} \frac{(1-u)^{m-1}(1-x)^{N-m}}{(1-u x)^{N-m+2}} \\
& \times \frac{e^{-\delta}}{K}{ }_{1} F_{1}\left(N-m+2,1 ; \frac{\delta x(1-u)}{1-x u}\right) d x .
\end{array}
$$

\section{B. NMF in non-Gaussian environment}

In the literature of radar detection and estimation, Spherically Invariant Random Vector (SIRV) modeling and Complex Elliptical Symmetric distributions (CES), originally introduced by Kelker in [16], have been considered and have been studied for their good statistical properties and for their good fitting to experimental nonGaussian radar data [17]. A good review on these distributions can be found in [8], [18]. A $m$-dimensional complex, zero-mean random vector follows a CES distribution if its PDF can be written as

$$
g_{\mathbf{c}}(\mathbf{c})=\left|\boldsymbol{\Sigma}^{-1}\right| h_{\mathbf{c}}\left(\mathbf{c}^{H} \boldsymbol{\Sigma}^{-1} \mathbf{c}\right),
$$

where $h_{\mathbf{c}}: \mathbb{R}^{+} \rightarrow \mathbb{R}^{+}$is any function such that (9) defines a PDF, $\boldsymbol{\Sigma}$ is a scatter matrix. It will be denoted $\mathbf{c} \sim \mathcal{C} \mathcal{E S}\left(\mathbf{0}_{\mathbf{m}}, \boldsymbol{\Sigma}\right)$. $\boldsymbol{\Sigma}$ reflects the structure of the $\mathrm{CM}$ of $\mathbf{c}$, i.e. the covariance matrix is equal to $\boldsymbol{\Sigma}$ up to a scale factor. One can notice that the Gaussian distribution is a particular case of CES. In this paper, we will assume that without loss of generality, $\boldsymbol{\Sigma}$ will be chosen equal to $\mathbf{M}$.

Due to the homogeneity of degree 0 of the NMF under $H_{0}$ hypothesis, the corresponding theoretical relationship between the detection threshold $\lambda$ and $P_{f a}=\mathbb{P}\left(H(\mathbf{M})>\lambda \mid H_{0}\right)$ is still given by $P_{f a}=(1-\lambda)^{m-1}$, even when the noise is CES distributed. When the cell under test is distributed according to any CES distributions, the corresponding relationship under $H_{1}$ hypothesis remains very difficult to obtain or even, to our knowledge, impossible to be derived.

When the cell under test contains SIRV distributed noise, the PDF of $p_{H(\mathbf{M})}$ of $H(\mathbf{M})$ under $H_{1}$ can be derived. When the cell under test contains SIRV noise, the noise $\mathbf{c}$ can be characterized by $\mathbf{c}=\sqrt{\tau} \mathbf{n}$ where $\mathbf{n}$ is a $m$-dimensional complex Gaussian vector $\mathcal{C N}(\mathbf{0}, \mathbf{M})$ and where $\tau$ is a positive scalar random variable and characterized by its PDF $p_{\tau}($.$) . Conditionally to this scalar random$ variable, the SNR is therefore given by $\alpha^{2} \mathbf{p}^{H} \mathbf{M}^{-1} \mathbf{p} / \tau$. We suppose in the following that $E[\tau]=1$ that implies that the final SNR is always given by $\delta=\alpha^{2} \mathbf{p}^{H} \mathbf{M}^{-1} \mathbf{p} / \sigma^{2}$. Conditionally to $\tau$, the PDF $p_{H(\mathbf{M})}$ of $H(\mathbf{M})$ under $H_{1}$ is given by (3) after replacing $\delta$ by $\delta / \tau$ and we obtain the final PDF :

$$
p_{H(\mathbf{M})}(u)=\int_{0}^{\infty} e^{-\delta / \tau} \beta_{1, m-1}(u)_{1} F_{1}\left(m, 1 ; \frac{u \delta}{\tau}\right) p_{\tau}(\tau) d \tau .
$$

The final $P_{d}-\lambda$ relationship of NMF test can be derived by simply integrating (10) with respect to $u$ from $\lambda$ to $\infty$.

$$
\begin{aligned}
P_{d}=1-\int_{0}^{+\infty} d \tau \int_{0}^{\lambda} e^{\delta(u-1) / \tau} \beta_{1, m-1}(u)_{1} F_{1} & \left(1-m, 1 ;-\frac{u \delta}{\tau}\right) \\
& \times p_{\tau}(\tau) d u .
\end{aligned}
$$

In the CES or SIRV distributed environment, the $M$-estimators are defined as the unique solution of the following equation

$$
\widehat{\mathbf{M}}=\frac{1}{N} \sum_{k=1}^{N} u\left(\mathbf{c}_{k}^{H} \widehat{\mathbf{M}}^{-1} \mathbf{c}_{k}\right) \mathbf{c}_{k} \mathbf{c}_{k}^{H},
$$

where $u($.$) stands for any real-valued function that satisfies a set of$ general assumptions (see [8], [9]), mainly for ensuring the existence, uniqueness and convergence of the previous equation. Note that MLEs are particular solutions of the previous equation. An attractive and powerful estimator, independent of the CES distribution, is the Tyler's estimator also called the Fixed Point and defined as the solution of:

$$
\widehat{\mathbf{M}}=\frac{m}{N} \sum_{k=1}^{N} \frac{\mathbf{c}_{k} \mathbf{c}_{k}^{H}}{\mathbf{c}_{k}^{H} \widehat{\mathbf{M}}^{-1} \mathbf{c}_{k}} \text {. }
$$

\section{ASYMPTOTIC BEHAVIOR OF THE ANMF TEST}

The goal of this section is to propose two different ways of deriving an approximate distribution of the test $H(\widehat{\mathbf{M}})$ built with any $M$-estimators under both $H_{0}$ and $H_{1}$ hypotheses and under Gaussian or non-Gaussian noise. The first approach consists in exploiting the asymptotic distribution of the $M$-estimators presented in previous section while the second approach is directly exploiting the asymptotic distribution of the asymptotic distribution of the ANMF built with any $M$-estimates.

A. Exploitation of the asymptotic behavior of the $M$-estimators For all $M$-estimator $\widehat{\mathbf{M}}$ which verifies equation (12), one has the important asymptotical statistical behavior:

$$
\sqrt{N}(\operatorname{vec}(\widehat{\mathbf{M}}-\mathbf{M})) \stackrel{d}{\longrightarrow} \mathcal{G C N}\left(\mathbf{0}_{m^{2}, 1}, \boldsymbol{\Sigma}_{M}, \boldsymbol{\Omega}_{M}\right),
$$




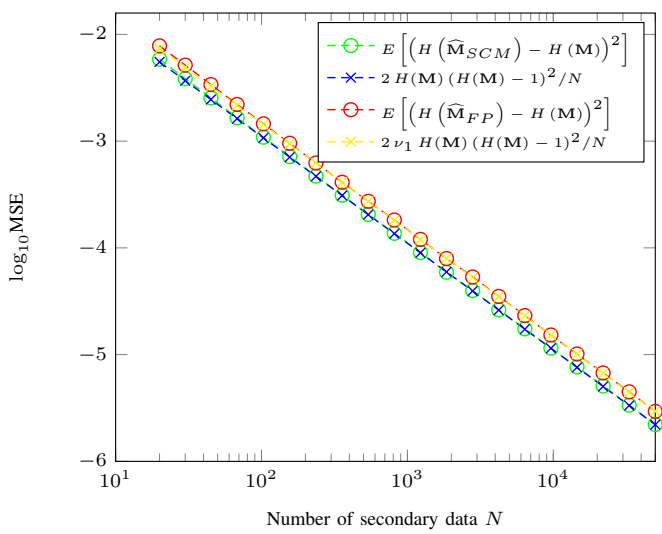

Fig. 1. Empirical variance of the ANMF built with the $\operatorname{SCM}\left(\nu_{1}=1\right)$ and Tyler's $M$-estimator $\left(\nu_{1}=(m+1) / m\right)$ in Gaussian environment and theoretical asymptotic variance for $m=3$ and $\mathbf{M}=\mathbf{I}_{3}$.

where $\mathbf{M}$ is the consistent limit of $\widehat{\mathbf{M}}$ and $\operatorname{GCN}\left(\mathbf{0}, \boldsymbol{\Sigma}_{M}, \boldsymbol{\Omega}_{M}\right)$ denotes the Generalized Complex Normal distribution with $\boldsymbol{\Sigma}_{M}$ the covariance matrix and $\boldsymbol{\Omega}_{M}$ the pseudo-covariance matrix defined as

$$
\begin{aligned}
& \boldsymbol{\Sigma}_{M}=\nu_{1} \mathbf{M}^{T} \otimes \mathbf{M}+\nu_{2} \operatorname{vec}(\mathbf{M}) \operatorname{vec}(\mathbf{M})^{H}, \\
& \boldsymbol{\Omega}_{M}=\nu_{1}\left(\mathbf{M}^{T} \otimes \mathbf{M}\right) \mathbf{K}+\nu_{2} \operatorname{vec}(\mathbf{M}) \operatorname{vec}(\mathbf{M})^{T},
\end{aligned}
$$

where $\mathbf{K}$ is the commutation matrix which transforms $\operatorname{vec}(\mathbf{A})$ into $\operatorname{vec}\left(\mathbf{A}^{T}\right), \nu_{1}$ and $\nu_{2}$ are real scalars relying on the CES distribution and given in [8], [9].

Let us first consider the two ANMF PDF given by (5) under $H_{1}$ hypothesis and (6) under $H_{0}$ hypothesis. Note that these two equations provide the exact distributions of $H\left(\widehat{\mathbf{M}}_{S C M}\right)$ under both $H_{0}$ and $H_{1}$ hypotheses when the secondary data are Gaussian distributed and for a cell under test containing also Gaussian noise. Now, for $N$ sufficiently large, equation (14) states that a $M$-estimator built with $N \nu_{1}$ observations behaves as the SCM built with $N$ observations. Consequently, combining this result with equations (5) and (6) or equivalently with equations (7) and (8), leads to the approximate distribution for $H(\widehat{\mathbf{M}})$ under both hypotheses where $\widehat{\mathbf{M}}$ stands for any $M$-estimator or for the Tyler's estimator.

Due to the homogeneity of degree 0 of the ANMF under $H_{0}$ hypothesis, the corresponding theoretical relationship between the detection threshold $\lambda$ and $P_{f a}=\mathbb{P}\left(H(\widehat{\mathbf{M}})>\lambda \mid H_{0}\right)$ is still given by the asymptotic corrected version of (7), i.e. when $N$ is replaced by $N / \nu_{1}$, even when the noise is CES distributed.

When the cell under test contains also SIRV or CES noise, the PDF of $p_{H(\mathbf{M})}$ of $H(\widehat{\mathbf{M}})$ under $H_{1}$ has to be derived. In the same way as in the previous section, we obtain therefore the final $P_{d}-\lambda$ relationship given in (8) by correcting $N$ with $N / \nu_{1}$, by setting $a=N-m+2$ and conditioning and integrating over the texture PDF $p_{\tau}$ :

$$
\begin{aligned}
P_{d}=1- & \int_{0}^{+\infty} d \tau \int_{0}^{1} d u \int_{0}^{\lambda} u^{a-1} \frac{(1-u)^{m-1}(1-x)^{a-2}}{(1-u x)^{a}} \\
& \times \frac{1}{K} e^{-\delta / \tau}{ }_{1} F_{1}\left(a, 1 ; \frac{\delta}{\tau} \frac{x(1-u)}{1-x u}\right) p_{\tau}(\tau) d x
\end{aligned}
$$

B. Exploitation of the asymptotic statistical behavior of the ANMF built with $M$-estimators
The asymptotic behavior of all the $M$-estimators can then be linked to the ANMF asymptotic statistical behavior thanks to the following result [13]:

$$
H(\widehat{\mathbf{M}}) \stackrel{d}{\longrightarrow} \mathcal{N}\left(H(\mathbf{M}), 2 \nu_{1} H(\mathbf{M})(H(\mathbf{M})-1)^{2} / N\right) .
$$

It is important to notice that the previous results are also valid for the SCM when the observations are Gaussian $\left(\nu_{1}=1, \nu_{2}=0\right)$, see e.g. [19]) and for the Tyler's estimator for CES-distributed observations $\left(\nu_{1}=(m+1) / m\right.$ and $\nu_{2}=-(m+1) / m^{2}$, e.g. [20]). The ANMF built with any $M$-estimators or with Tyler's estimator behaves asymptotically as ANMF built with SCM, it differs only from the scalar quantities $\nu_{1}$ and $\nu_{2}$. Notice that the previous asymptotic distribution is a distribution conditional to the observation $\mathbf{y}$ that appears in $H(\mathbf{M})$. Consequently, a supplementary step is required to obtain the asymptotic distribution of $H(\widehat{\mathbf{M}})$.

The figure 1 illustrates the result (17) when comparing, in Gaussian environment and for different number $N$ of secondary data, the empirical variance of $H\left(\widehat{\mathbf{M}}_{S C M}\right)$ with the theoretical variance given in (17) for $\nu_{1}=1$ and $m=3$ and the empirical variance of $H\left(\widehat{\mathbf{M}}_{F P}\right)$ with the theoretical variance given by (17) for $\nu_{1}=(m+1) / m$ for $m=3$.

When the cell under test contains Gaussian, SIRV noise or CES noise, the PDF $p_{H(\mathbf{M})}$ of $H(\mathbf{M})$ under $H_{0}$ is still given by (3) because of the homogeneity of the function $H($.) by CES or SIRV noise family. According to the result given by (17), for $N$ large enough, considering that $H(\widehat{\mathbf{M}}) \sim \mathcal{N}\left(X, 2 \nu_{1} X(X-1)^{2} / N\right)$ where $X \sim p_{H(\mathbf{M})}$, one can obtain the asymptotic distribution $f_{H(\widehat{\mathbf{M}})}^{a}$ of $H(\widehat{\mathbf{M}})$ under $H_{0}$ hypothesis:

$$
f_{H(\widehat{\mathbf{M}})}^{a}(u)=\int_{0}^{1} \frac{\sqrt{N} \exp \left(-\frac{N(u-x)^{2}}{4 \nu_{1} x(x-1)^{2}}\right)}{\sqrt{4 \pi \nu_{1} x(x-1)^{2}}} p_{H(\mathbf{M})}(x) d x .
$$

where $p_{H(\mathbf{M})}($.$) is given by (3). Now, if we denote \Phi($.$) the cumula-$ tive distribution of the Normal distribution, one obtains respectively the corresponding asymptotical $P_{f a}-\lambda$ relationship:

$$
P_{f a}=1-\int_{0}^{1} \beta_{1, m-1}(x) \Phi\left(\frac{\sqrt{N}(\lambda-x)}{\sqrt{2 \nu_{1} x(x-1)^{2}}}\right) d x .
$$

Under $H_{1}$ hypothesis and only for any SIRV distributed noise, the PDF of $p_{H(\mathbf{M})}$ of $H(\mathbf{M})$ has been derived and is given by (10), leading to the final expression of the asymptotic distribution $f_{H(\widehat{\mathbf{M}})}^{a}(u)$ of $H(\widehat{\mathbf{M}})$ :

$$
\begin{aligned}
f_{H(\widehat{\mathbf{M}})}^{a}(u) & =\int_{0}^{\infty} d \tau \int_{0}^{1} \frac{\sqrt{N} \exp \left(-\frac{N(u-x)^{2}}{4 \nu_{1} x(x-1)^{2}}\right)}{\sqrt{4 \pi \nu_{1} x(x-1)^{2}}} \\
& \times e^{-\delta / \tau} \beta_{1, m-1}(u)_{1} F_{1}\left(m, 1 ; \frac{u \delta}{\tau}\right) p_{\tau}(\tau) d x
\end{aligned}
$$

The final Probability of Detection expression if then obtained by evaluating $P_{d}=\mathbb{P}\left(H(\widehat{\mathbf{M}})>\lambda \mid H_{1}\right)$ :

$$
\begin{gathered}
P_{d}=1-\int_{0}^{\infty} p_{\tau}(\tau) d \tau \int_{0}^{1} \beta_{1, m-1}(x) e^{\delta(x-1) / \tau} \\
\times{ }_{1} F_{1}\left(1-m, 1 ;-x \frac{\delta}{\tau}\right) \Phi\left(\frac{\sqrt{N}(\lambda-x)}{\sqrt{2 \nu_{1} x(x-1)^{2}}}\right) d x
\end{gathered}
$$

Under $H_{1}$ hypothesis, the previous developments are not valid for any CES distributed cell under test because this latter can be written 
as $\mathbf{y}=\alpha \mathbf{p}+\tau \mathbf{u}=\alpha \mathbf{p}+\tau \mathbf{x} /\|\mathbf{x}\|$. We can remark that $\mathbf{x}$ and $\tau /\|\mathbf{x}\|$ are not independent. The conditioning approach proposed in this paper is hence not at all valid.

\section{Simulations}

In this section, we set the Toeplitz covariance matrix $\mathbf{M}$ whose entries are defined as $M_{i j}=\rho^{|i-j|}$ where $\rho=0.5$. The noise in the cell under test is $\mathrm{K}$-distributed with the shape parameter $\nu=0.5$. Figures 2 and 3 show, for $P_{f a}=10^{-3}, m=10$ and two numbers of secondary data $N$, the different $P_{d}$-SNR relationship for the NMF given by (11), the first approximate distribution of the ANMF built with Tyler's estimator (16), the asymptotic expression derived in (21) for the Tyler's estimator and the empirical $P_{d}$ for the TylerANMF. The asymptotic regime is achieved and one can observe a good agreement between the two asymptotic distributions derived in this paper.

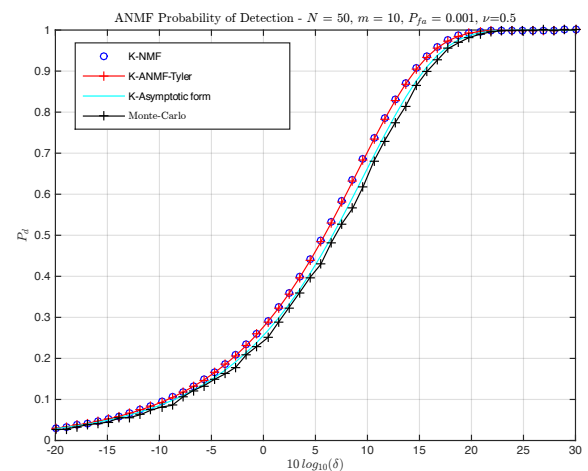

Fig. 2. Comparison between $P_{d}$ and SNR $\delta$ relationships for the NMF, the ANMF built with Tyler's estimator and its asymptotic form, $m=10$, $N=50, \nu_{1}=1.1$ and $P_{f a}=10^{-3}, \mathbf{p}=[1, \ldots, 1]^{T}, \mathbf{y}=\alpha \mathbf{p}+\mathbf{c}$ where $\mathbf{c} \sim K_{\nu}$ where $K_{\nu}$ is a multivariate K-distribution with shape parameter $\nu=0.5$ and covariance matrix $\mathbf{M}$

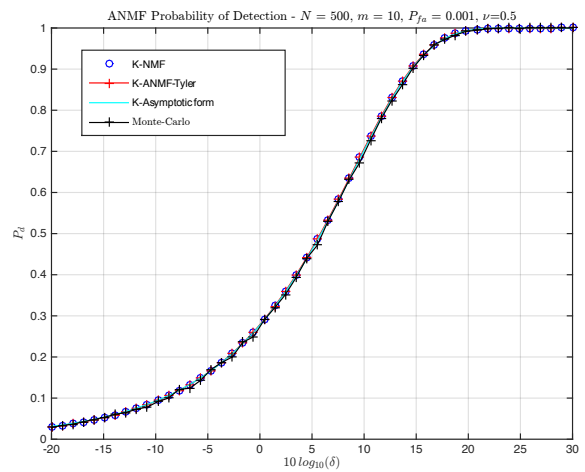

Fig. 3. Comparison between $P_{d}$ and SNR $\delta$ relationships for the NMF, the ANMF built with Tyler's estimator and its asymptotic form, $m=10$, $N=500, \nu_{1}=1.1$ and $P_{f a}=10^{-3}, \mathbf{p}=[1, \ldots, 1]^{T}, \mathbf{y}=\alpha \mathbf{p}+\mathbf{c}$ where $\mathbf{c} \sim K_{\nu}$ where $K_{\nu}$ is a multivariate $\mathrm{K}$-distribution with shape parameter $\nu=0.5$ and covariance matrix $\mathbf{M}$

\section{CONCLUSION}

In the context of robust detection in Gaussian or non-Gaussian noise, two asymptotic distributions of the ANMF have been proposed for both $H_{0}$ and $H_{1}$ hypotheses. More precisely, using robust covariance matrix estimators such as $M$-estimators or the Tyler's estimator, two asymptotic approximations of the corresponding ANMF distribution have been derived following different approaches. First, we have combined the exact distribution of the ANMF built with the SCM under Gaussian noise and the asymptotic properties of the robust estimators. Finally, we have directly derived the asymptotic distribution of the robust ANMF under CES environment. These results provide a very good approximation of the ANMF distribution even for a small number of observations and have been applied to theoretically regulate the false alarm probability and to evaluate the detection performance.

\section{REFERENCES}

[1] F. Gini, "Sub-Optimum Coherent Radar Detection in a Mixture of KDistributed and Gaussian Clutter," IEE Proc. on Radar, Sonar Navig., vol. 144, pp. 39-48, February 1997.

[2] S. Kraut and L. L. Scharf, "The CFAR adaptive subspace detector is a scale-invariant GLRT," Signal Processing, IEEE Transactions on, vol. 47, no. 9, pp. 2538-2541, 1999.

[3] F. C. Robey, D. R. Fuhrmann, E. J. Kelly, and R. Nitzberg, "A CFAR adaptive matched filter detector," Aerospace and Electronic Systems, IEEE Transactions on, vol. 28, no. 1, pp. 208-216, January 1992.

[4] E. J. Kelly, "An adaptive detection algorithm," Aerospace and Electronic Systems, IEEE Transactions on, no. 2, pp. 115-127, 1986.

[5] E. Conte and M. Lops and G. Ricci, "Asymptotically Optimum Radar Detection in Compound-Gaussian Clutter," IEEE Transactions on Aerospace and Electronic Systems, vol. 31, no. 2, pp. 617-625, 1995.

[6] P. J. Huber, "Robust estimation of a location parameter," The Annals of Mathematical Statistics, vol. 35, no. 1, pp. 73-101, 1964.

[7] R. A. Maronna, "Robust $M$-estimators of multivariate location and scatter," Annals of Statistics, vol. 4, no. 1, pp. 51-67, January 1976.

[8] E. Ollila, D. E. Tyler, V. Koivunen, and H. V. Poor, "Complex elliptically symmetric distributions: Survey, new results and applications," Signal Processing, IEEE Transactions on, vol. 60, no. 11, pp. 5597 -5625 , nov. 2012.

[9] M. Mahot, F. Pascal, P. Forster, and J.-P. Ovarlez, "Asymptotic properties of robust complex covariance matrix estimates," Signal Processing, IEEE Transactions on, vol. 61, no. 13, pp. 3348-3356, July 2013.

[10] F. Pascal, Y. Chitour, J.-P. Ovarlez, P. Forster, and P. Larzabal, "Covariance structure maximum-likelihood estimates in compound Gaussian noise: existence and algorithm analysis," Signal Processing, IEEE Transactions on, vol. 56, no. 1, pp. 34-48, Jan. 2008.

[11] D. Tyler, "A distribution-free $m$-estimator of multivariate scatter," The Annals of Statistics, vol. 15, no. 1, pp. 234-251, 1987.

[12] L. L. Scharf and D. W. Lytle, "Signal detection in Gaussian noise of unknown level: an invariance application," Information Theory, IEEE Transactions on, vol. 17, pp. 404-411, July 1971.

[13] F. Pascal and J.-P. Ovarlez, "Asymptotic Properties of the Robust ANMF," in IEEE International Conference on Acoustics, Speech, and Signal Processing, ICASSP-15, Brisbane, Australia, April 2015.

[14] S. Kraut, L. Scharf, and L. M. Whorter, "Adaptive Subspace Detector," Signal Processing, IEEE Transactions on, vol. 49, pp. 1-16, January 2001.

[15] F. Pascal, J.-P. Ovarlez, P. Forster, and P. Larzabal, "Constant false alarm rate detection in spherically invariant random processes," in Proc. of the European Signal Processing Conf., Vienna, September 2004, pp. 21432146.

[16] D. Kelker, "Distribution theory of spherical distributions and a locationscale parameter generalization," Sankhyā: The Indian Journal of Statistics, Series A, vol. 32, no. 4, pp. 419-430, 1970.

[17] E. Conte and A. De Maio, "Mitigation techniques for non-Gaussian sea clutter," Oceanic Engineering, IEEE Journal of, vol. 29, no. 2, pp. 284-302, 2004.

[18] G. Frahm, "Generalized elliptical distributions: Theory and applications," Ph.D. dissertation, Universität zu Köln, 2004

[19] T. W. Anderson, An Introduction to Multivariate Statistical Analysis, 1st ed. John Wiley \& Sons, New York, 1958.

[20] F. Pascal, P. Forster, J.-P. Ovarlez, and P. Larzabal, "Performance analysis of covariance matrix estimates in impulsive noise," Signal Processing, IEEE Transactions on, vol. 56, no. 6, pp. 2206-2217, Jun. 2008. 